PROCEEDINGS OF THE

AMERICAN MATHEMATICAL SOCIETY

Volume 139, Number 3, March 2011, Pages 849-854

S 0002-9939(2010)10547-6

Article electronically published on August 13, 2010

\title{
SMOOTH PI ALGEBRAS WITH FINITE DIVISOR CLASS GROUP
}

\author{
A. BRAUN AND C. R. HAJARNAVIS
}

(Communicated by Martin Lorenz)

\begin{abstract}
We have shown in an earlier paper that the divisor class group of the centre of a smooth PI algebra with trivial $K_{0}$ is a torsion group of finite exponent. We show here that this group need not be finite even in the affine case. Our example is an Azumaya algebra of global dimension 2. We also provide a positive result in a special case.
\end{abstract}

\section{INTRODUCTION}

Throughout this paper, all rings are associative with identity and all modules are unitary. Unless otherwise stated, all modules considered are right $R$-modules.

Our basic objects of study are smooth PI algebras. These are Noetherian PI rings of finite global dimension, having the additional property that the projective dimension of all maximal two-sided ideals belonging to a given clique is the same.

The investigation of these rings has been carried out over the last three decades under different perspectives. They were first termed 'moderated algebras' in a somewhat less well-known paper by Vasconcelos [13]. They were later described as 'homologically homogeneous' (hom. hom., for short) in a paper by Brown and Hajarnavis [2]. More recently, Stafford and Zhang [12] have used the term 'smooth' giving the concept a geometrical flavour. However, as the term smooth has several meanings, some authors (e.g. [11]) have preferred to retain the name homologically homogeneous.

A recent development in this area is the description of properties of the centres of smooth PI algebras. These results were partially inspired by papers in noncommutative geometry. A recent result of Stafford and Van den Bergh [1] states that in characteristic zero, the centre must have rational singularity. Our interest in the centre is related to the properties of its divisor class group [1]. The smooth $\mathrm{PI}$ rings we discuss in [1] are more restrictive and satisfy the 'trivial $K_{0}$ ' property. This means that every finitely generated projective (left as well as right) module whose Goldie rank is a multiple of the Goldie rank of the ring, must be stably free. This property holds trivially in local commutative rings and is satisfied by several smooth PI algebras.

It should be mentioned that smooth PI algebras are always semi-prime and integral over their centres [12]. Consequently, in the affine case they are actually finite modules over their Noetherian centres.

Received by the editors June 1, 2009 and, in revised form, April 14, 2010.

2010 Mathematics Subject Classification. Primary 16E65, 16H05, 16P50, 16R20.

(C)2010 American Mathematical Society Reverts to public domain 28 years from publication 
The basic question we address here is as follows.

Question 1.1. Let $R$ be a smooth Noetherian prime PI algebra affine over a field $F$ and with trivial $K_{0}$. Let $Z(R)$ be the centre of $R$. Then is the divisor class group $\mathrm{Cl}(Z(R))$ a finite group?

The motivation for such a question comes from the following;

Theorem $1.2([1$, Theorem $\mathrm{A}])$. Let $R$ be as above. Then $C l(Z(R))$ is a d-torsion group, where $d$ is the PI degree of $R$.

The following result serves as positive evidence for the validity of the above question.

Theorem 1.3 ([1, Theorem D]). Let $R$ be a connected graded Noetherian PI algebra of finite global dimension and affine over a field $F$ of characteristic 0 . Then $\mathrm{Cl}(Z(R))$ is a finite group.

The proof of this theorem is rather complicated and uses deep results of Danilov which in turn depend on Hironaka's resolution of singularity theorem. Thus the charF $=0$ assumption seems essential. The purpose of this paper is twofold. Firstly, we present an additional positive result.

Theorem 1.4. Let $R$ be as in Question 1.1 and let $Q(R)$ denote the quotient ring of $R$. Assume, in addition, that $Q(R)=M_{d}(Z(Q(R)))$, where $d$ is the PI degree of $R$ and $M_{d}(-)$ represents the $d \times d$ matrix ring. Then $C l(Z(R))$ is a finite group.

In view of this it is rather surprising that we are able to obtain a negative answer to Question 1.1 in the case when $\operatorname{char} F=p>0$.

Example 1.5. Let $p$ be a prime number. There exists an affine PI algebra $T$ over a field $F$ with $\operatorname{char} F=p>0$ such that $T$ has the following properties:

(i) $T$ is an Azumaya algebra with gl.dim.T $=2$.

(ii) $T$ is a noncommutative integral domain, and every finitely generated projective $T$-module is stably free.

(iii) Every height one prime ideal of $T$ is principal but is not necessarily generated by a central element.

(iv) $C l(Z(T))$ is an infinite torsion group and the exponent $p$ of this group is also the PI degree of $T$.

(v) In particular, $T$ is a smooth PI algebra with trivial $K_{0}$.

Thus the above provides a counterexample to Question 1.1. It is unclear if 2 is the minimum possible dimension for such an example. However, when the field in question is algebraically closed, we show that this is indeed the case (Proposition 2.2).

\section{Proof of Theorem 1.4}

Our first step is a reduction to Azumaya algebras. By the Artin-Procesi theorem [10. Theorem 1.8.33] we may pick a central element $\delta \in Z(R)$ such that $R\left[\frac{1}{\delta}\right]$ is Azumaya. Clearly,

$$
Z\left(R\left[\frac{1}{\delta}\right]\right)=Z(R)\left[\frac{1}{\delta}\right] .
$$


Therefore by Nagata's theorem [4, Theorem 7.1] we have the following short exact sequence:

$$
0 \rightarrow K \rightarrow C l(Z(R)) \rightarrow C l\left(Z(R)\left[\frac{1}{\delta}\right]\right) \rightarrow 0,
$$

where $K$ is generated by all height one prime ideals $p$ in $Z(R)$ with $\delta \in p$.

Now by [2] $Z(R)$ is a Krull domain. Consequently, by [6, p. 16] this set of prime ideals is finite. However by Theorem 1.2 each such $p$ represents a $d$-torsion element in $C l(Z(R))$ implying that $K$ is a finite group.

The properties which $R\left[\frac{1}{\delta}\right]$ inherits from $R$ are as follows. By [1, Lemma 10], the $\operatorname{ring} R\left[\frac{1}{\delta}\right]$ is a smooth PI algebra. Let $N$ be a finitely generated right projective $R\left[\frac{1}{\delta}\right]$ module whose Goldie rank, $\operatorname{rank} N$, equals $\operatorname{rankR}$. Consequently, after applying a right $R$-isomorphism, we may assume that $N \subseteq Q(R)$, the quotient ring of $R$. After multiplying by a central element of $Z(R)$ one may think of $N$ as a right ideal of $R\left[\frac{1}{\delta}\right]$, and therefore $N=M\left[\frac{1}{\delta}\right]$ for some right ideal $M$ of $R$ such that $\operatorname{rank} M=\operatorname{rankR}$. So since $N$ is, in addition, $R\left[\frac{1}{\delta}\right]$-projective, then $N$ is stably free by [1, Corollary 8].

One could show the same for a projective module $N$ whose rank is a multiple of the rank of $R$. But we have no need for it here.

So by a change of notation, we assume that $R$ is a smooth Azumaya algebra whose projective modules of rank equal to that of $R$ are stably free. Now since $R$ is an Azumaya algebra, $R$ is a finitely generated projective $Z(R)$-module [3, Theorem 3.4]. Moreover, $Z(R)$ is a $Z(R)$-direct summand of $R$ [3, Lemma 3.1]. Consequently, by [5, Theorem 7.2.8], gl.dim. $R<\infty$ implies that gl.dim. $Z(R)<\infty$; that is, $Z(R)$ is a regular ring. Consequently, $C l(Z(R))=\operatorname{Pic}(Z(R))$, where Pic denotes the Picard group.

Recall that $Q(R)=M_{d}(Z(Q(R)))$ by assumption. Let $\left\{e_{i j} \mid 1 \leq i, j \leq d\right\}$ be a set of matrix units of $Q(R)$ and choose $\lambda \in Z(R)$ such that $e_{i j} \in R\left[\frac{1}{\lambda}\right]$. Therefore,

$$
M_{d}\left(Z(R)\left[\frac{1}{\lambda}\right]\right) \subseteq R\left[\frac{1}{\lambda}\right]
$$

Both terms here are Azumaya algebras with the same centre $Z(R)\left[\frac{1}{\lambda}\right]$ and possess the same PI degree $d$. Consequently, by [3, Cor. 3.6, p. 54], $M_{d}\left(Z(R)\left[\frac{1}{\lambda}\right]\right)=R\left[\frac{1}{\lambda}\right]$ and as in the previous argument, we may also assume that $R=M_{d}(Z(R))$, in addition to being Azumaya, is also smooth and is a ring over which each projective module whose rank equals that of $R$ is stably free. We shall finish the proof by showing that $\operatorname{Pic}(Z(R))=0$.

Let $I \subseteq Z(R)$ be a projective ideal. Then $(I, \cdots, I)$ ( $d$ times) is a right $R=M_{d}(Z(R))$-module. Moreover since $(I, \cdots, I)(d$ times $)$ is a projective $Z(R)$ module, the lifting property of $R / Z(R)$ [3. Prop. 2.3, p. 48] shows that $(I, \cdots, I)$ ( $d$ times) is a projective right $R=M_{d}(Z(R)$ )-module. Consider the $d \times d$ matrix

$$
N \equiv\left[\begin{array}{ccccc}
I & \cdot & \cdot & \cdot & I \\
Z(R) & \cdot & \cdot & \cdot & Z(R) \\
\cdot & \cdot & \cdot & \cdot & \cdot \\
\cdot & \cdot & \cdot & \cdot & \cdot \\
Z(R) & \cdot & \cdot & \cdot & Z(R)
\end{array}\right] .
$$


Thus $N$ is a direct sum of $d$ projective right $R$-modules. Therefore $N_{R}$ is projective. Moreover, since $\operatorname{rank} N=\operatorname{rank} R$, the module $N_{R}$ is right stably free. Hence for some positive integer $k$,

$$
N \oplus M_{d}(Z(R)) \oplus \cdots \oplus M_{d}(Z(R)) \cong M_{d}(Z(R)) \oplus \cdots \oplus M_{d}(Z(R)),
$$

where there are $k$ terms on each side of the above right $R$-module isomorphism. Hence $N \oplus R \oplus \cdots \oplus R=y_{1} R+\cdots+y_{k} R$, where $y_{i} \in N \oplus R \oplus \cdots \oplus R$. Write $\check{y}_{i}$ for $y_{i}$ in column form. Then

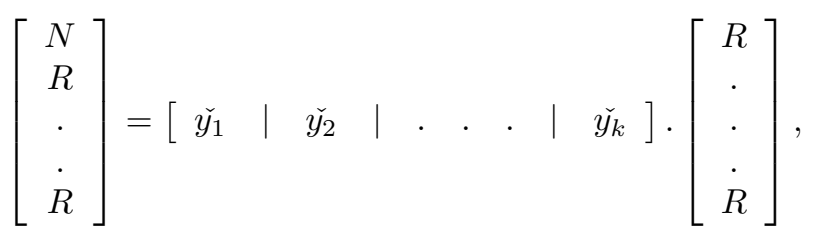

where the columns are $k \times 1$ matrices.

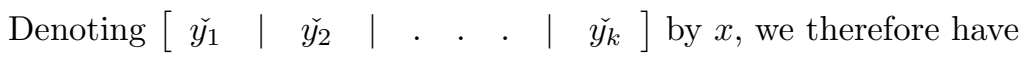

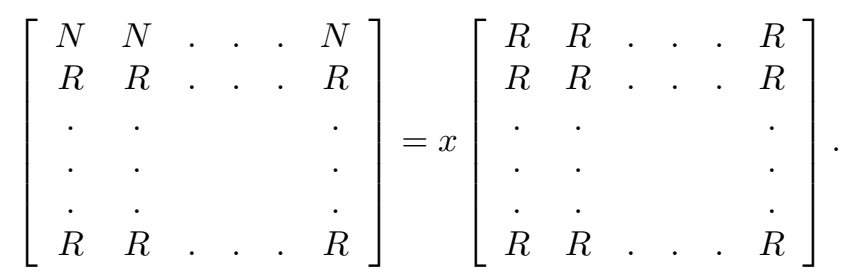

Here the matrices are of size $k \times k$. As a $k d \times k d$ matrix over $Z(R)$ the left hand side of $(*)$ has the first row consisting of $I$ 's and the other rows of $Z(R)$ 's. Therefore,

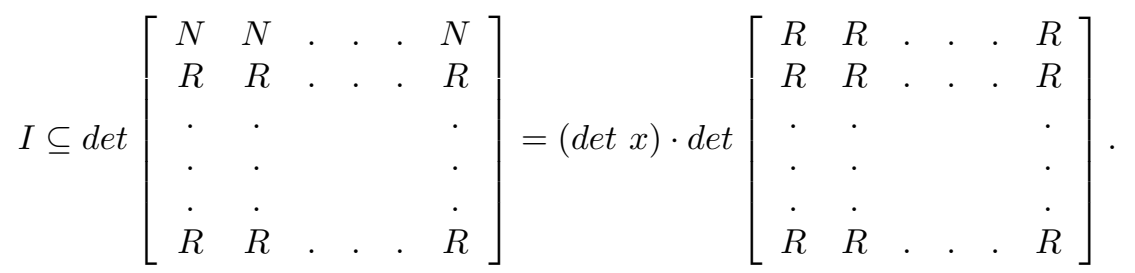

Therefore $I \subseteq \operatorname{det}(x) Z(R)$. Now since

$$
x \in\left[\begin{array}{cccccc}
N & N & \cdot & \cdot & \cdot & N \\
R & R & \cdot & \cdot & \cdot & R \\
\cdot & \cdot & & & \cdot \\
\cdot & \cdot & & & \cdot \\
\cdot & \cdot & & & \cdot \\
R & R & \cdot & \cdot & \cdot & R
\end{array}\right]
$$

we have $\operatorname{det}(x) \in I$. This gives us $I=\operatorname{det}(x) Z(R)$, and the result follows.

Remark 2.1. The ring $R\left[\frac{1}{\delta}\right]$ inherits the trivial $K_{0}$ assumption from $R$; if this trivial $K_{0}$ assumption is a Morita invariant property, then $Z(R)\left[\frac{1}{\delta}\right]$ will also have it since $R\left[\frac{1}{\delta}\right]=M_{d}\left(Z(R)\left[\frac{1}{\delta}\right]\right)$. Hence $I$ being projective over $Z(R)\left[\frac{1}{\delta}\right]$ would be stably free and hence free. However, we do not know this at the outset.

The next result is a consequence of Theorem 1.4 . 
Proposition 2.2. Suppose that $R$ is a smooth affine Noetherian prime PI algebra over a field $F$. Assume in addition that:

(i) $K \cdot \operatorname{dim} R=1$,

(ii) $R$ has trivial $K_{0}$ and

(iii) $F$ is algebraically closed.

Then $C l(Z(R))$ is a finite group.

Proof. Since $R$ is a finite $Z(R)$-module, we have $K \cdot \operatorname{dim} Z(R)=1$. Hence $Q(Z(R))$ is an algebraic extension of $F(t)$ for some transcendental element $t$ over $F$. Consequently, by Tsen's theorem [7, Theorem 8.4] we have $Q(R)=M_{d}(Q(Z(R))$. The result now follows by Theorem 1.4 .

\section{A counterexample}

As shown in 14 there exist two affine Dedekind domains $R$ and $S$ such that $R \subset S, \operatorname{char} R=p>0, S=H\left[z, z^{-1}\right]$, where $H$ is a field, $z$ is a variable, $S=$ $R\langle x\rangle=R+R x+\cdots+R x^{p-1}$ with $x^{p} \in R$. Also there exists a derivation $\mathcal{D}$ on $S$ such that $\mathcal{D}(x)=1$ and $\mathcal{D}(R)=0$. Consequently, $S^{\mathcal{D}}=R$.

Observe that $C l(S)=0$ and moreover every finitely generated projective $S$ module is stably free since $S=H\left[z, z^{-1}\right]$ and $H$ is a field. More importantly, $C l(R)$ is a $p$-torsion group but not a finite group. Let $T=S[\xi, \mathcal{D}]$ be the skew polynomial ring (or Ore extension) with variable $\xi$. So $[\xi, a]=\mathcal{D}(a)$ for all $a \in S$. Let $\Lambda \subset R$ be some subfield. Then $\Lambda \mathcal{D}$ is a 1-dimensional Lie algebra and $T \cong S * \mathbf{U}(\Lambda \mathcal{D})$ in the notation of [5, 1.7.12].

We shall now verify the properties of $T$ in Example 1.5

(i) $Z(T)=R\left[\xi^{p}\right]$ and since $C l(R) \cong C l\left(R\left[\xi^{p}\right]\right)$ by [4, p.36], we conclude that $C l(Z(T))$ is infinite.

(ii) $R$ is affine and therefore $T$ is affine over a central subfield. Moreover, $T$ is an Azumaya algebra of rank $p^{2}$ over its centre. To see this, observe that $1, \xi, \xi^{2}, \cdots, \xi^{p-1}$ is a basis of $T$ over $S\left[\xi^{p}\right]$. Also $\operatorname{rank}_{R} S=p$. Hence $\operatorname{rank}_{R\left[\xi^{p}\right]} S\left[\xi^{p}\right]=p$ and $\operatorname{rank}_{Z(T)} T=p^{2}$. In particular, PI.deg $T=p$. Now $[\xi, x]=\mathcal{D}(x)=1$. Hence the subring $\Lambda\{\xi, x\}$ is a homomorphic image of $\mathcal{A}_{1}$, the first Weyl algebra over $\Lambda$. Since by $9 \mathcal{A}_{1}$ is an Azumaya algebra of rank $p^{2}$, this subring is also an Azumaya algebra of rank $p^{2}$ over its centre. It follows by [10, Theorem 1.8.48], using the fact that a central polynomial has value 1, that $T$ is an Azumaya algebra of rank $p^{2}$ over its centre.

(iii) gl.dim.T $=2$ : This follows easily from [5, 7.7.5(ii) or 7.5.3(i)] since gl.dim. $S=1$.

(iv) By the above, $T$ is smooth, being an Azumaya algebra of finite global dimension. Moreover, since $T \cong S * \mathbf{U}(\Lambda \mathcal{D})$ and since every finitely generated projective $S$-module is stably free, the same can be seen to hold for $T$ by applying [5, 12.3.3(ii)]. In particular $T$ has trivial $K_{0}$. Consequently, by Walker's theorem [8, Theorem 10.4.13], the ring $T$ must be a (non-commutative) integral domain.

(v) Every height one prime ideal in $T$ is principal (but is not necessarily generated by a central element). Indeed, let $P$ be a height one prime ideal of $T$ and let $I=P \cap S\left[\xi^{p}\right]$. Clearly $I \neq\{0\}$. If $I$ is a reflexive ideal in $S\left[\xi^{p}\right]$, then $I=a S\left[\xi^{p}\right]$ for some $a \in I$ by the UFD property of $S\left[\xi^{p}\right]$.

Now $T$ is an Azumaya algebra and in particular $P=(P \cap Z(T)) T$. Since $P \cap Z(T) \subseteq P \cap S\left[\xi^{p}\right]=I$, we obtain that $P=a T$, as claimed. 
It remains, therefore, to show that $I$ is reflexive. Let $q=P \cap Z(T)$. By going down [10, Theorem 4.4.24] we have that height $(q)=1$. Now $S\left[\xi^{p}\right]_{q}$ is a semi-local Dedekind domain and therefore we have $\left(I_{q}\right)^{* *}=I_{q}$. Now $I^{* *} \subseteq\left(I_{q}\right)^{* *} \cap S\left[\xi^{p}\right]=I_{q} \cap S\left[\xi^{p}\right]$. So we only need to show that $I_{q} \cap S\left[\xi^{p}\right]=I$.

Since $I_{q} \subseteq P_{q}$, we have $I_{q} \cap S\left[\xi^{p}\right] \subseteq P_{q} \cap T=P$. Hence $I_{q} \cap S\left[\xi^{p}\right] \subseteq$ $P \cap S\left[\xi^{p}\right]=I$.

\section{REFERENCES}

1. A. Braun and C. R. Hajarnavis, Smooth polynomial identity algebras with almost factorial centers, J. Algebra 299 (2006) 124-150. MR2225768 (2007f:16055)

2. K. A. Brown and C. R. Hajarnavis, Homologically homogeneous rings, Trans. Amer. Math. Soc. 281 (1984) 197-208. MR0719665 (85e:16046)

3. F. Demeyer and E. Ingraham, Separable algebras over commutative rings, Lecture Notes in Math., no. 181, Springer, Berlin, 1971. MR0280479 (43:6199)

4. R. M. Fossum, The divisor class group of a Krull domain, Springer, Berlin, 1973. MR0382254 $(52: 3139)$

5. J. C. McConnell and J. C. Robson, Non-commutative Noetherian rings, Wiley-Interscience, New York, 1987. MR0934572 (89j:16023)

6. H. Matsumura, Commutative ring theory, Cambridge Studies in Adv. Math. 8, Cambridge University Press, New York, 1989. MR.1011461 (90i:13001)

7. M. Orzech and C. Small, The Brauer group of commutative rings, Marcel Dekker, Inc., New York, 1975. MR0457422(56:15627)

8. D. S. Passman, The algebraic structure of group rings, Wiley, New York, 1977. MR0470211 (81d:16001)

9. P. Revoy, Algèbres de Weyl en caractéristique p. C. R. Acad. Sci. Paris Sér. A-B 276 (1973), A225-A228. MR0335564 (49:345)

10. L. H. Rowen, Polynomial identities in ring theory, Academic Press, New York, 1980. MR.0576061 (82a:16021)

11. J. T. Stafford and M. Van den Bergh, Non-commutative resolutions and rational singularities, Michigan Math. J. 57 (2008) 659-674. MR2492474 (2010d:14002)

12. J. T. Stafford and J. J. Zhang, Homological properties of (graded) Noetherian PI rings, J. Algebra 168 (1994) 888-1026. MR1293638 (95h:16030)

13. W. V. Vasconcelos, On quasi-local regular algebras, Symposia Mathematica, Vol. XI (Convegno di Algebra Commutativa, INDAM, Rome, 1971), pp. 11-22. Academic Press, London, 1973. MR 330159 (48:8497)

14. B. Wajnryb, Divisor class group descent for affine Krull domains, J. Algebra 93 (1985) 150156. MR772476 (86a:13004)

Department of Mathematics, University of Haifa, Haifa 31905, Israel

E-mail address: abraun@math2.haifa.ac.il

Mathematics Institute, University of Warwick, Coventry CV4 7AL, England

E-mail address: crh@maths.warwick.ac.uk 